\title{
Discussion on "Prediction of ground vibrations resulting from the blasting operations in an open-pit mine by adaptive neuro-fuzzy inference system"
}

\author{
Tarkan Erdik
}

Received: 11 January 2008/Accepted: 3 April 2008/Published online: 18 April 2008

(C) Springer-Verlag 2008

The discusser appreciates the exhaustive study undertaken by the authors for predicting ground vibrations resulting from the blasting operations in an open-pit mine by adaptive neuro-fuzzy inference system. However, the present paper has some scientific mistakes especially in the basic conception and application of the fuzzy theory. The straightforward application of ANFIS through software gives rise to physical and logical problems even though the results may seem convenient from the numerical tests point of view. In any scientific research the first steps should include the logical and physical principles of the data at hand in addition to its simple treatment through some graphs such as the scatter diagrams prior to classical regression or ANFIS methodology application. Otherwise, complete dependence on any methodology especially by software without knowing its internal domain may lead to erroneous scientific applications. The paper by the authors suffers from such defects even though it seems numerically sound. The following logical points may be raised on the scientific grounds.

1. Membership functions (MFs) both in Figs. 6 and 7 are misleading and they are not physically and logically plausible. For instance in Fig. 7, "VH" and "H" MFs are almost equivalent within say, $\pm 2 \%$ error limits. It implies that the same set of data portion is attached with two different fuzzy sets, which cannot be acceptable. This is due to the fact that the authors of this paper as many all over the world enter the available data to ANFIS program especially in MATLAB software and

T. Erdik $(\square)$

Hydraulics Division, Faculty of Civil Engineering, Istanbul Technical University, Maslak, 34469 Istanbul, Turkey

e-mail: erdik@itu.edu.tr get the results automatically without any further plausible assessment. Therefore, the trained model is impratical. The ANFIS model constructed by the authors should be trained with three membership functions for the input variable, "charge weight per delay (W)", in order to have a parsimonious model. The authors also failed to consider this point in the modeling.

2. In Fuzzy Logic (FL) principle, the most left and right MFs should have membership degree equal to 1 (Sen 2004, 2007), otherwise they cannot be linguistically correct. However, the left-most MFs, namely, "VC" and " $\mathrm{C}$ " for the input variable "Distance (D)" in Fig. 6 are decreasing limbs towards "low" values. In addition, the right-most MFs, namely "VF" for the input variable "Distance (D)" in Fig. 6 and "H" and "VH" for the input variable "Charge weight per delay (W)" in Fig. 7 are decreasing limbs towards "high" values, which are contrary to FL principle. For instance, how could 1000 have less membership degree than 800 as "VH" in Fig. 7. Similar reasoning applies also for "H" in Fig. 7 in addition to "VF" and "VC" fuzzy sets in Fig. 6.

Acknowledgments The author is especially indebted to Prof. Dr. Zekai Sen for his useful suggestions.

\section{References}

Sen Z (2004) Fuzzy logic and system models in water sciences. Turkish Water Foundation, Istanbul

Sen Z (2007) Discussion Takagi-Sugeno fuzzy inference system for modeling stage-discharge relationship. J Hydrol 337:242-243 